\title{
Note
}

\section{Tea Catechins Inhibit Cell Proliferation Through Hydrogen Peroxide- Dependent and -Independent Pathways in Human T lymphocytic Leukemia Jurkat Cells}

\author{
Yue $\mathrm{TANG}^{1,3}$, Naomi $\mathrm{AbE}^{2}$, Hang $\mathrm{QI}^{3}$, Beiwei Zhu ${ }^{3}$, Yoshiyuki Murata ${ }^{2}$ and Yoshimasa NaKAmurA ${ }^{2 *}$ \\ ${ }^{I}$ Graduate School of Natural Science and Technology, Okayama University, Okayama 700-8530, Japan \\ ${ }^{2}$ Graduate School of Environmental and Life Science, Okayama University, Okayama 700-8530, Japan \\ ${ }^{3}$ School of Food Science and Technology, Dalian Polytechnic University, Dalian 116034, China
}

Received May 8, 2014 ; Accepted July 22, 2014

In the present study, we investigated the anti-proliferative effects of four tea catechins: (-)-epicatechin (EC), (-)-epigallocatechin (EGC), (-)-epicatechin gallate (ECg) and (-)-epigallocatechin gallate (EGCg) in human T lymphocytic leukemia Jurkat cells. Each catechin induced a significant cytotoxic effect on Jurkat cells. Combinations of EGCg with other catechins additively potentiated anti-proliferation, extracellular hydrogen peroxide formation, c-Jun N-terminal kinase (JNK) activation and interferon (IFN)- $\gamma$ mRNA expression. Catalase partly but significantly abolished the cytotoxicity induced by EGC or EGCg, whereas it did not influence the EC- or ECg-induced effect. Among the tea catechins, ECg synergistically enhanced the EGCg-induced JNK phosphorylation and IFN- $\gamma$ expression. The present findings provide evidence that tea catechins are able to concertedly induce cytotoxicity through the JNK/IFN- $\gamma$ pathway in both a hydrogen peroxide-dependent and -independent manner.

Keywords: tea catechins, hydrogen peroxide, cytotoxicity, c-Jun N-terminal kinase, interferon- $\gamma$, jurkat cells

\section{Introduction}

Green tea serves as a rich source of polyphenol antioxidants, particularly tea catechins such as (-)-epicatechin (EC), (-)-epigallocatechin (EGC), (-)-epicatechin gallate (ECg) and (-)-epigallocatechin gallate (EGCg). EGCg is the major catechin in green tea, and accounts for $50-80 \%$ of total tea catechins (Harbowy et al., 1997). Although EGCg is an effective antioxidant and scavenger of reactive oxygen species (ROS), it can undergo autooxidation and behave as a pro-oxidant (Yang et al., 2002). EGCg induces the activation of stress signals, such as mitogen activated protein kinases (MAPKs), resulting in apoptosis via hydrogen peroxide-dependent mechanisms in several cancer cells (Nakagawa et al., 2004; Chan et al., 2006). Extracellular hydrogen peroxide formation and c-Jun N-terminal kinase (JNK), one of the MAPKs, are known to contribute to EGCg-induced cytotoxicity in human leukemia cells (Nakagawa et al., 2004; Wu et al., 2008). Also, we recently demonstrated that $\mathrm{EGCg}$ ameliorates photodynamic therapy responses against $\mathrm{T}$ lymphocytic leukemia cells via JNK activation and T cell-specific interferon- $\gamma$ (IFN- $\gamma$ ) expression (Qi et al., 2014).

In addition to $\mathrm{EGCg}$, other tea catechins including $\mathrm{EC}, \mathrm{EGC}$, and ECg also have pro-oxidative properties, and thus show antiproliferative effects on several cancer cell lines (Yang et al., 2002). For example, tea catechins inhibit the growth of human lung cancer PC-9 cells, with the order of potency being EGCg $=$ ECg $>$ EGC > EC (Okabe et al., 1997). Based on these data, EGCg has been viewed as the most important compound to explain the actual anticancer potential of green tea. However, the same group suggested 
that the total green tea extract has stronger effects than the EGCg content alone would have indicated (Okabe et al., 1997). These results led us to assume that the constituents of green tea other than EGCg have combinatory effects on the anti-cancer activity. Although EGCg is reported to show a cytotoxic effect in human T lymphocytic leukemia cells (Wu et al., 2008), the impact of other catechins or their combinatory effects with EGCg remains unclear.

Because all tea catechins have pro-oxidative properties, we hypothesized that they would induce cytotoxic effects in $\mathrm{T}$ lymphocytes in a hydrogen peroxide-dependent manner. To test this hypothesis, we analyzed the influence of tea catechins individually and in combination with EGCg on the proliferation of human T lymphocytic leukemia Jurkat cells. Since these cells share many properties with native $\mathrm{T}$ lymphocytes, they are an appropriate model to analyze the effects of chemicals on T cells (Abraham and Weiss, 2004; Murugaiyan et al., 2013). Furthermore, the extracellular hydrogen peroxide formation and JNK-dependent expression of IFN- $\gamma$ were assessed, because these are the potential determinants for apoptosis induction in T lymphocytes ( $\mathrm{Wu}$ et al., 2008; Qi et al., 2012; Qi et al., 2014).

\section{Materials and Methods}

Human Jurkat T lymphocytes (RIKEN Cell Bank; Tsukuba, Japan) were maintained in RPMI-1640 medium and supplemented with $10 \%(\mathrm{v} / \mathrm{v})$ fetal bovine serum (FBS), $50 \mathrm{U} / \mathrm{mL}$ of penicillin and $50 \mu \mathrm{g} / \mathrm{mL}$ of streptomycin at $37^{\circ} \mathrm{C}$ under $5 \% \mathrm{CO}_{2}$ and $95 \%$ air. For experiments, cells were seeded in complete medium and treated with EC, EGC, ECg (Sigma, St., Louis, MO, USA), EGCg (Wako, Osaka, Japan) or vehicle (methanol or dimethylsulfoxide; DMSO, final $0.2 \%, \mathrm{v} / \mathrm{v}$ ).

To determine hydrogen peroxide formation in the medium, we performed a FOX assay as previously reported (Qi et al., 2011).

Cell viability was evaluated using a trypan blue exclusion assay as previously reported (Qi et al., 2014). Jurkat cells $\left(1 \times 10^{6}\right.$ cells $\left./ \mathrm{mL}\right)$ were treated with catechins individually and in combination with EGCg in serum-free RPMI-1640 medium in 6-well plates for $1 \mathrm{~h}$ at $37^{\circ} \mathrm{C}$, washed with fresh medium, and incubated for an additional $23 \mathrm{~h}$ in serum-free RPMI-1640 medium. The cells were collected by centrifugation and resuspended in PBS(-). Each cell suspension was mixed with 0.4\% trypan blue stain. The viable cells (those that excluded blue dye) were counted with a hemocytometer under a light microscope (Nikon, Tokyo, Japan).

JNK phosphorylation was determined by western blotting as previously reported (Qi et al., 2011). Phosphorylated JNK (p-JNK) antibody was purchased from Cell Signaling Technology (Beverly, MA). The antibody to actin (Santa Cruz, CA, USA) was used as the loading control.

The IFN- $\gamma$ mRNA level was measured by reverse transcription polymerase chain reaction (RT-PCR) analysis. Total RNA $(5 \mu \mathrm{g})$ was reverse transcribed with OligodT to cDNA using M-MLV reverse transcriptase (Takara, Kyoto, Japan). PCR amplification was then performed with Taq polymerase (Takara) and specific primers. The forward and reverse primers and the expected PCR product sizes are as follows: I F N- $\gamma$ 5' - G G T C A T T C A G A T G T A G C G G A - 3' a n d 5'-GCGTTGGACATTCAAGTCAG-3' (270 bp); $\beta$-actin 5 ' - G T C A C C C A C A C T G T G C C C A T C T A - 3' a n d 5'-GCAATGCCAGG GTACATGGTGGT-3' (455 bp). The annealing temperature used in this study was $58^{\circ} \mathrm{C}$ for IFN- $\gamma$ and $65^{\circ} \mathrm{C}$ for $\beta$-actin. The PCR products were then subjected to agarose gel electrophoresis (2\%), stained with ethidium bromide, and photographed. Densitometric analysis of the bands was carried out using the MultiGauge software program (Fujifilm, Tokyo, Japan).

All values are expressed as means $\pm \mathrm{SD}$. Data were analyzed by a one-way analysis of variance (ANOVA) followed by multiple comparisons among means (Tukey's HSD) using XLSTAT software version 2014.3.04 (Addinsoft, Paris, France). Different letters above the bars indicate significant differences among treatments for each compound $(P<0.05)$.

\section{Results and Discussion}

The maximum absorption of tea catechins occurs at around 1-2 h (Van het Hof et al., 1998; Miyazawa et al., 2000) and the elimination half-life is also very fast (Scalbert and Williamson, 2000). To clearly compare the cytotoxicity of each tea catechin alone, Jurkat cells were treated with $100 \mu \mathrm{M}$ of each catechin in the serum-free medium for $1 \mathrm{~h}$, washed with fresh medium, and then incubated for an additional $23 \mathrm{~h}$. Each catechin treatment significantly decreased the viability of Jurkat cells, with the order of potency being $\mathrm{EGC}>\mathrm{EGCg}>>\mathrm{ECg}=\mathrm{EC}$ (Fig. 1). This result

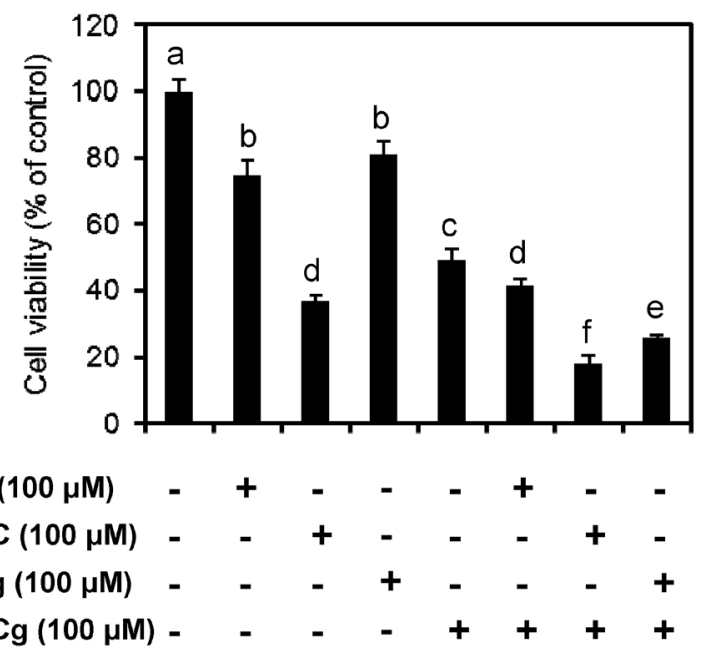

Fig. 1. Effect of tea catechins on the viability of Jurkat cells. Jurkat cells $\left(1 \times 10^{6}\right.$ cells $\left./ \mathrm{mL}\right)$ were treated with catechins $(100 \mu \mathrm{M})$ individually or in combination with EGCg for $1 \mathrm{~h}$ at $37^{\circ} \mathrm{C}$, washed with fresh medium and then incubated for another $23 \mathrm{~h}$. The cell viability was determined by a trypan blue dye exclusion assay. The values represent the means $\pm \mathrm{SD}$ of three separate experiments. Bars with the same letters are not significantly different at $P<0.05$. 
is consistent with a previous paper demonstrating the cytotoxic effects of tea catechins in Chinese hamster V79 cells (Nakamaya et al., 2002). To determine the effect of tea catechin combinations on Jurkat cell proliferation, the cells were then co-incubated with EGCg plus EC, EGC or ECg. Each tea catechin in combination with $\mathrm{EGCg}$ showed greater cytotoxicity than $\mathrm{EGCg}$ alone. The order of effectiveness was EGC + EGCg $(82 \%)>\mathrm{ECg}+\mathrm{EGCg}$ $(74 \%)>\mathrm{EC}+\mathrm{EGCg}(59 \%)$. These data implied that $\mathrm{ECg}$ is more effective for combination use with EGCg than EC, even though the effect of ECg alone is quite similar to that of EC.

We previously reported that the EGCg-induced cytotoxicity in Jurkat cells was significantly inhibited by catalase (Wu et al., 2008). Thus, to clarify the mechanism underlying the tea catechininduced cytotoxicity, hydrogen peroxide levels in the media were determined. As shown in Fig. 2, incubation of the medium with each tea catechin $(10 \mu \mathrm{M})$ significantly increased the hydrogen peroxide level in the rank order of $\mathrm{EGCg}>\mathrm{EGC}>\mathrm{ECg}>\mathrm{EC}$. This effect of each catechin is stoichiometric (data not shown). The coexistence of EC, EGC or ECg with EGCg apparently enhanced hydrogen peroxide formation compared to EGCg alone, suggesting that other tea catechins can additively increase the hydrogen peroxide level in combination with EGCg. To investigate whether hydrogen peroxide is involved in the catechin-induced cytotoxicity, catalase $(1000 \mathrm{U} / \mathrm{mL})$ was used as the hydrogen peroxide scavenger. As shown in Fig. 3, catalase partly but significantly abolished the cytotoxicity induced by EGC or EGCg, whereas it did not influence the EC- or ECg-induced effect. These results suggested that each tea catechin inhibits cell proliferation, at least partly, through a hydrogen peroxide-independent pathway.

We determined the effects of tea catechins on the expression of p-JNK and IFN- $\gamma$ mRNA, because these are the potential
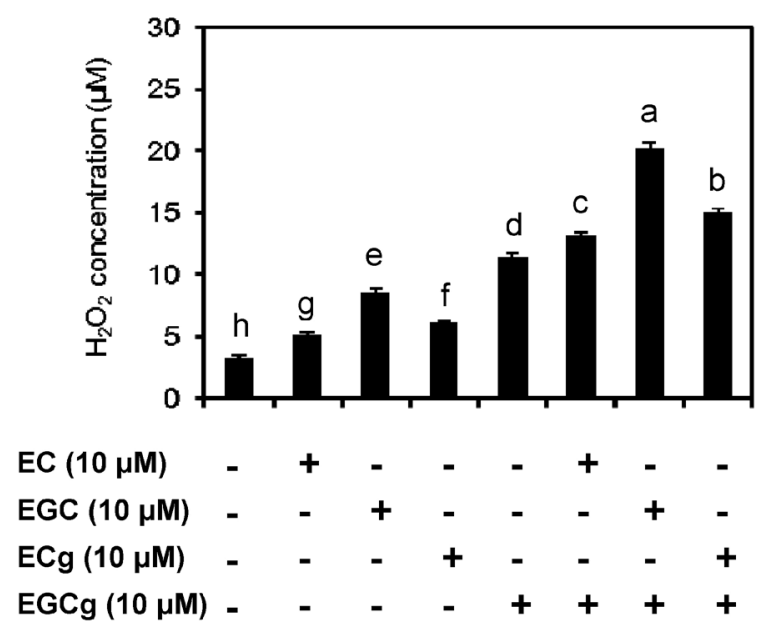

Fig. 2. Extracellular hydrogen peroxide formation by tea catechins in the cell culture medium. Tea catechins $(10 \mu \mathrm{M})$ individually or in combination with EGCg were added to RPMI 1640 serum-free medium for $1 \mathrm{~h}$ at $37^{\circ} \mathrm{C}$. The hydrogen peroxide concentration was measured by a FOX assay. The values represent the means \pm SD of three separate experiments. Bars with the same letters are not significantly different at $P<0.05$. determinants for the hydrogen peroxide-dependent apoptosis induction in T lymphocytes (Qi et al., 2012; Qi et al., 2014). As shown in Fig. 4, EGC as well as EGCg showed a significant increase in the p-JNK level in Jurkat cells, whereas neither EC nor $\mathrm{ECg}$ enhanced it. EGCg in combination with $\mathrm{EC}, \mathrm{EGC}$ or $\mathrm{ECg}$ markedly potentiated JNK phosphorylation compared to those induced by EGCg alone. This tendency is consistent with the result of IFN- $\gamma$ gene expression (Fig. 5).

It should be noted that the enhancing effects of $\mathrm{ECg}$ on the EGCg-induced expression of $\mathrm{p}-\mathrm{JNK}$ and IFN- $\gamma$ are synergistic, even though the hydrogen peroxide level in the $\mathrm{ECg}+\mathrm{EGCg}$ group was much less than that in the EGC + EGCg group (Fig. 2). These results implied that ECg enhances the $\mathrm{p}-\mathrm{JNK} / \mathrm{IFN}-\gamma$ pathway, possibly through an extracellular hydrogen peroxide-independent pathway.

The present results demonstrate that green tea catechins solely and concertedly induce anti-proliferative effects in human $\mathrm{T}$ lymphocytic leukemia Jurkat cells. Hydrogen peroxide upregulates the expression of double-stranded RNA-activated protein kinase and IFN- $\gamma$ to induce apoptosis in Jurkat cells (Garg et al., 2005). We have also reported that a JNK inhibitor, SP600125, partly but significantly inhibited IFN- $\gamma$ expression as well as apoptosis induced by EGCg in Jurkat cells (Wu et al., 2008; Wu et al., 2009). Furthermore, an IFN- $\gamma$ signaling inhibitor, AG490, significantly inhibited hydrogen peroxide-dependent cytotoxicity (Qi et al., 2012). Although it is difficult to rule out the involvement of other redox-sensitive signals in catechin-induced cytotoxicity, tea catechins might potentiate the EGCg-induced cytotoxicity by enhancing the $\mathrm{T}$ cell-specific JNK/IFN- $\gamma$ pathway. Although the tested concentration $(100 \mu \mathrm{M})$ is much higher than those observed in blood or tissues (usually lower than $1 \mu \mathrm{M}$ ) after the ingestion of

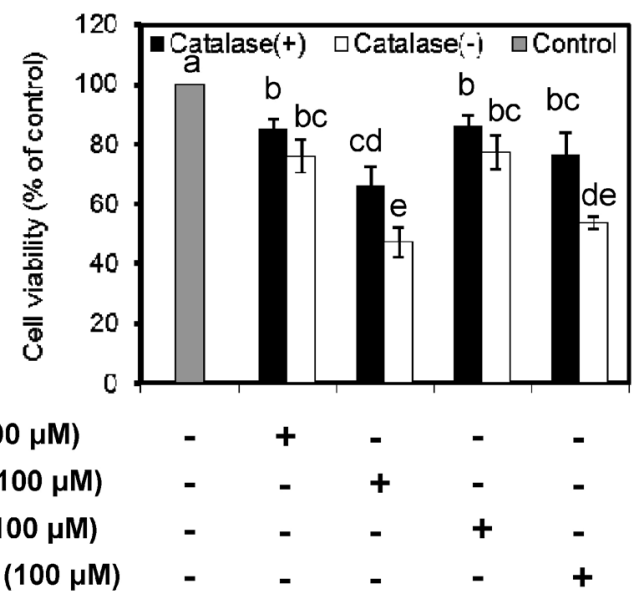

Fig. 3. Effect of catalase on tea catechin-induced cytotoxicity in Jurkat cells. Jurkat cells $\left(1 \times 10^{6}\right.$ cells $\left./ \mathrm{mL}\right)$ were pretreated with or without catalase $(1000 \mathrm{U} / \mathrm{mL})$ for $30 \mathrm{~min}$, and stimulated with catechins $(100 \mu \mathrm{M})$ for $1 \mathrm{~h}$. The cells were incubated with fresh serum-free medium for an additional $23 \mathrm{~h}$. Each bar represents the mean $\pm \mathrm{SD}(\mathrm{n}=$ 3 ). Bars with the same letters are not significantly different at $P<0.05$. 


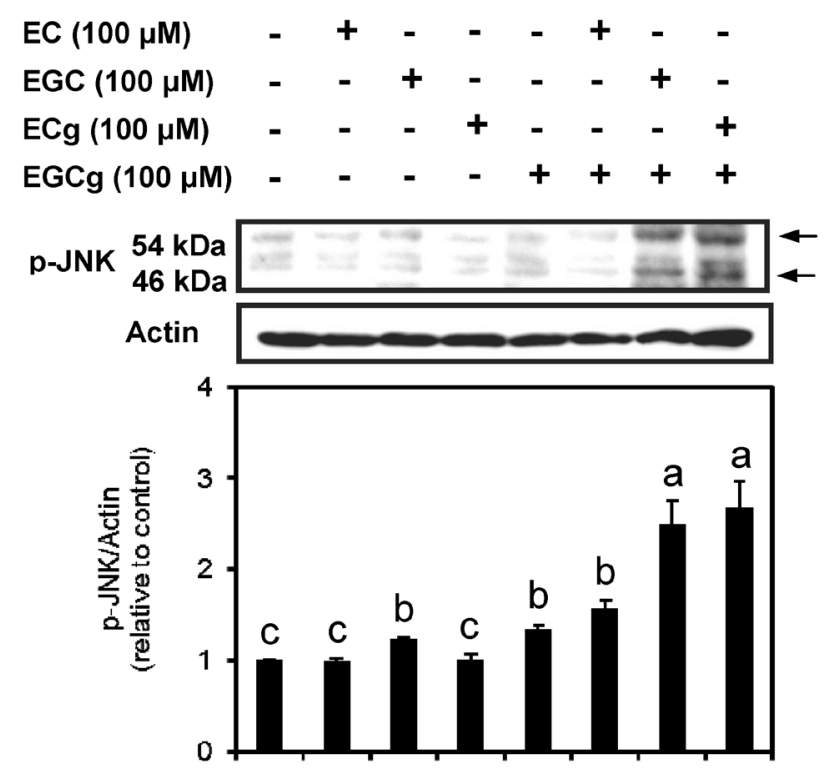

Fig. 4. Effect of tea catechins on JNK phosphorylation in Jurkat cells. Jurkat cells $\left(1 \times 10^{6}\right.$ cells $\left./ \mathrm{mL}\right)$ were treated with tea catechins $(100 \mu \mathrm{M})$ individually or in combination with EGCg for $1 \mathrm{~h}$ at $37^{\circ} \mathrm{C}$ and then harvested. The JNK phosphorylation was analyzed by western blotting. The values represent the means $\pm \mathrm{SD}$ of three separate experiments. Bars with the same letters are not significantly different at $P<0.05$.

tea by humans (Kim et al., 2000), lower concentrations of tea catechins can also enhance the EGCg-induced cytotoxic effect (data not shown). Since $10 \mu \mathrm{M}$ EGCg is able to enhance photodynamic therapy responses against $\mathrm{T}$ lymphocytic leukemia cells through the JNK/IFN- $\gamma$ pathway (Qi et al., 2014), the tea catechin mixture is also applicable not only as a therapeutic or preventative agent, but also as an ameliorating agent for photodynamic therapy against T-cell mediated skin diseases such as cutaneous T-cell lymphoma and psoriasis.

The rank order of cytotoxic activity in Jurkat cells was EGC > $\mathrm{EGCg} \gg \mathrm{ECg}=\mathrm{EC}$ (Fig. 1), which differed from the order of the hydrogen peroxide formation (Fig. 2). Although EGC or EGCg can induce cytotoxicity in a hydrogen peroxide-dependent manner, the hydrogen peroxide-independent pathway is involved in cytotoxicity induced by individual catechins (Fig. 3). Consistent with these data, the cytotoxicity of EGCg in Jurkat cells is not fully ascribed to hydrogen peroxide formation (Wu et al., 2008). The EGCginduced p-JNK and IFN- $\gamma$ expression is also partly inhibited by catalase (Wu et al., 2009). Although much less hydrogen peroxide was formed during the incubation of ECg than EGC or EGCg (Fig. 2), ECg showed significant cytotoxicity (Fig. 1). This tendency is consistent with a previous report (Nakayama et al., 2002). These results suggest that hydrogen peroxide formation is not the only factor governing the cytotoxicity of ECg. Nakayama and colleagues previously pointed out that the higher affinity of ECg for lipid bilayers compared to other catechins might also contribute to their cytotoxicity to some degree (Nakayama et al., 2002). Together, the finding that ECg synergistically enhanced the JNK/IFN- $\gamma$ pathway
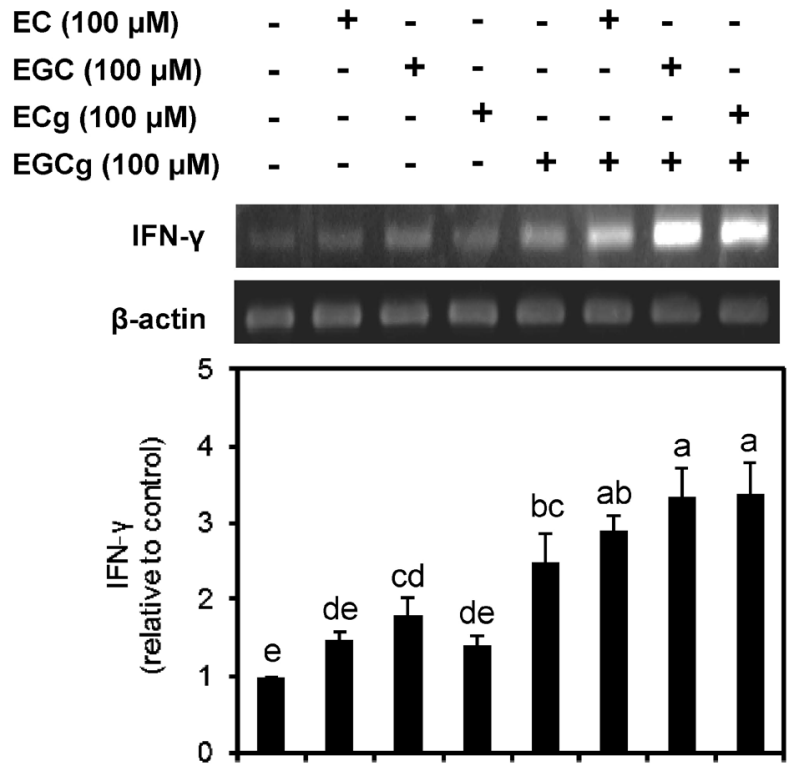

Fig. 5. Effect of tea catechins on IFN- $\gamma$ gene expression in Jurkat cells. Jurkat cells $\left(1 \times 10^{6}\right.$ cells $\left./ \mathrm{mL}\right)$ were treated with tea catechins $(100 \mu \mathrm{M})$ individually or in combination with EGCg for $1 \mathrm{~h}$ at $37^{\circ} \mathrm{C}$, washed with fresh medium and then incubated for another $2 \mathrm{~h}$. The IFN- $\gamma$ mRNA expression was analyzed by a RT-PCR assay. The values represent the means $\pm \mathrm{SD}$ of three separate experiments. Bars with the same letters are not significantly different at $P<0.05$.

(Fig. 4 and Fig. 5) led us to the assumption that intracellularlyaccumulated ECg is involved in EGCg-induced signal transduction. Future efforts will be concerned with the in vivo biological significance of the effect of tea catechin combinations as well as further elucidation of the signaling pathway of ECg-induced signal transduction.

Acknowledgments This study was supported by a grant-in-aid from the Ministry of Education, Culture, Sports, Science, and Technology of the Japanese Government (No. 25292073).

\section{References}

Abraham, R.T. and Weiss, A. (2004). Jurkat T cells and development of the T-cell receptor signalling paradigm. Nat. Rev. Immunol., 4, 301-308.

Chan, M.M., Soprano, K.J., Weinstein, K., and Fong, D. (2006).

Epigallocatechin-3-gallate delivers hydrogen peroxide to induce death of ovarian cancer cells and enhances their cisplatin susceptibility. J. Cell. Physiol., 207, 389-396.

Harbowy, M.E. and Balentine, D.A. (1997). Tea chemistry. Crit. Rev. Plant. Sci., 16, 415-480.

Garg, A.K., Buchholz, T.A., and Aggarwal, B.B. (2005). Chemosensitization and radiosensitization of tumors by plant polyphenols. Antioxid. Redox Signal., 7, 1630-1647.

Kim, S., Lee, M.J., Hong, J., Li, C., Smith, T.J., Yang, G.Y., Seril, D.N., and Yang, C.S. (2000). Plasma and tissue levels of tea catechins in rats and mice during chronic consumption of green tea polyphenols. Nutr. Cancer, 37, 41-48.

Miyazawa, T. (2000). Absorption, metabolism and antioxidative effects of 
tea catechin in humans. Biofactors, 13, 55-59.

Murugaiyan, J., Rockstroh, M., Wagner, J., Baumann, S., Schorsch, K., Trump, S., Lehmann, I., Bergen, M., and Tomm, J.M. (2013). Benzo[ $\alpha$ ] pyrene affects Jurkat $\mathrm{T}$ cells in the activated state via the antioxidant response element dependent Nrf2 pathway leading to decreased IL-2 secretion and redirecting glutamine metabolism. Toxicol. Appl. Pharmacol., 269, 307-316.

Nakagawa, H., Hasumi, K., Woo, J.T., Nagai, K., and Wachi, M. (2004). Generation of hydrogen peroxide primarily contributes to the induction of $\mathrm{Fe}(\mathrm{II})$-dependent apoptosis in Jurkat cells by (-)-epigallocatechin gallate. Carcinogenesis, 25, 1567-1574.

Nakayama, T., Ichiba, M., Kuwabara, M., Kajiya, K., and Kumazwa, S. (2002). Mechanisms and structural specificity of hydrogen peroxide formation during oxidation of catechins. Food Sci. Technol. Res., 8, 261270 .

Okabe, S., Suganuma, M., Hayashi, M., Sueoka, E., Komori, A., and Fujiki, H. (1997). Mechanisms of growth inhibition of human lung cancer cell line, PC-9, by tea polyphenols. Jpn. J. Cancer Res., 88, 639-643.

Qi, H., Takano, H., Kato, Y., Wu, Q., Ogata, C., Zhu, B.W., Murata, Y., and Nakamura, Y. (2011). Hydrogen peroxide-dependent photocytotoxicity by phloxine B, a xanthene-type food colorant. Biochim. Biophys. Acta., 1810, 704-712.
Qi, H., Zhu, B.W., Abe, N., Shin, Y., Murata, Y., and Nakamura, Y. (2012). Involvement of intracellular oxidative stress-sensitive pathway in phloxine B-induced photocytotoxicity in human T lymphocytic leukemia cells. Food Chem. Toxicol., 50,1841-1847.

Qi, H., Abe, N., Zhu, B.W., Murata, Y., and Nakamura, Y. (2014). (-)-Epigallocatechin-3-gallate ameliorates photodynamic therapy responses in an in vitro T lymphocyte model. Phytother. Res., in press.

Scalbert, A. and Williamson, G. (2000). Dietary intake and bioavailability of polyphenols. J. Nutr., 130, 2073-2085.

Van het Hof, K.H., Kivits, G.A.A., Weststrate, J. A., and Tijburg, L.B.M. (1998). Bioavailability of catechins from tea: the effect of milk. Eur. J. Clin. Nutr., 52, 356-359.

Wu, H., Yokoyama, T., Zhu, B., Shimoishi, Y., Murata, Y., and Nakamura, Y. (2008). (-)-Epigallocatechin-3-gallate potentiates the cytotoxicity induced by benzyl isothiocyanate and hydrogen peroxide in human Jurkat T lymphocytes. Biosci. Biotechnol. Biochem., 72, 3034-3037.

Wu, H., Zhu, B., Shimoishi, Y., Murata, Y., and Nakamura, Y. (2009). (-)-Epigallocatechin-3-gallate induces up-regulation of Th1 and Th2 cytokine genes in Jurkat T cells. Arch. Biochem. Biophys., 483, 99-105.

Yang, C.S., Maliakal, P., and Meng, X. (2002). Inhibition of carcinogenesis by tea. Annu. Rev. Pharmacol. Toxicol., 42, 25-54. 